\title{
Defense mechanism responses to COVID-19
}

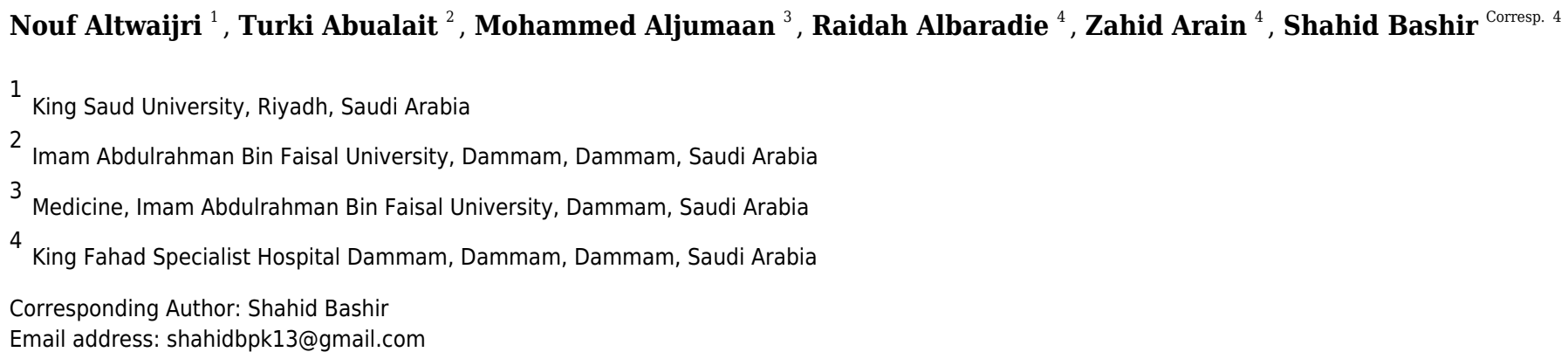

The COVID-19 pandemic has had a wide range of negative physical and mental impacts. This review begins with a theoretical explanation of the psychological defense mechanisms used to deal with the pandemic. It then discusses different categories of defense mechanisms and their roles in managing the impacts of psychological distress. The aim of this review is to highlight the various psychological defense mechanisms individuals use to deal with the pandemic and to discuss how adjustment mechanisms can protect individuals from internal and external threats by shielding the integrity of the ego (the mind) and helping individuals maintain their self-schema . 


\section{Review work}

\section{Defense mechanism responses to COVID-19}

3 Nouf Altwaijri', Turki Abualait ${ }^{2}$, Mohammed Aljumaan ${ }^{3}$, Raidah Albaradie ${ }^{4}$, Zahid Arain ${ }^{5}$,

4 Shahid Bashir ${ }^{*}$

$5 \quad{ }^{1}$ College of Medicine, King Saud University, Riyadh, Saudi Arabia

$6{ }^{2}$ College of Applied Medical Sciences, Imam Abdulrahman Bin Faisal University, Dammam,

7 Saudi Arabia.

$8{ }^{3}$ College of Medicine, Imam Abdulrahman Bin Faisal University, Dammam, Saudi Arabia.

$9{ }^{4}$ Neuroscience Center, King Fahad Specialist Hospital, Dammam, Saudi Arabia.

$10{ }^{5}$ Liver Transplant Department, King Fahad Specialist Hospital, Dammam, Saudi Arabia.

12 Running title: Defense mechanism and COVID-19

13

$14 *$ Corresponding author

15 Shahid Bashir

16 Neuroscience Center, King Fahad Specialist Hospital,

17 Ammar Bin Thabit Street, Dammam - 31444, Saudi Arabia.

18 E-mail address: shahidbpk13@gmail.com

19 Contact number: $+966-538152268$ 


\section{Abstract}

27 The COVID-19 pandemic has had a wide range of negative physical and mental impacts. This

28 review begins with a theoretical explanation of the psychological defense mechanisms used to

29 deal with the pandemic. It then discusses different categories of defense mechanisms and their

30 roles in managing the impacts of psychological distress. The aim of this review is to highlight the

31 various psychological defense mechanisms individuals use to deal with the pandemic and to

32 discuss how adjustment mechanisms can protect individuals from internal and external threats by

33 shielding the integrity of the ego (the mind) and helping individuals maintain their self-schema.

35 Key words: Covid-19, Defense mechanism, Pandemic 


\section{Introduction}

50 The COVID-19 pandemic has had serious negative impacts on the physical and mental health of

51 individuals around the world ${ }^{1}$. Several reports, on the general population, have shown that the

52 psychological adverse effects of contagion and government-enforced quarantines, during the

53 pandemic, extends afar the fear of viral infection and comprises social separation impacts,

54 feeling helpless, loss of freedom, and uncertainty of disease progression ${ }^{2,3}$. Such effects are

55 associated with internalizing (anxious, depressive symptoms, social withdraws, and somatic

56 complaints) and externalizing (oppositional, aggressive and violent/delinquent) psychological

57 behavior that might be developed intensely ${ }^{4}$. These psychological behavior can be evolved

58 dramatically into life-threatening consequences such as suicide ideation and action ${ }^{5}$.

59 Furthermore, evidences clearly have shown that frontline healthcare workers, who were closely

60 involved in diagnosing, medically managing and treating infected patients with COVID-19 virus,

61 in addition to witnessing the suffering and death of patients during the pandemic were highly

62 likely to evolve psychological distress and mental health impairments ${ }^{6,7,8}$. These impairments

63 including a wide range of non-specific symptoms of anxiety, distress, fear, depression, and

64 insomnia, might ultimately trigger long-lasting and clinically significant personal and psychiatric

65 disorders $9,10,11,12$.

66 Given the fact that the COVID-19 pandemic has negative influences on psychological well-

67 being, it is crucial to further explore the human behaviors that are usually elicited by the

68 psychological distress. The pandemic's effects reach beyond the viral infection and consist of

69 complex psychodynamic processes that continuously impact individuals' behavior, emotions,

70 and life satisfaction ${ }^{13}$. 
71 In behavioral science, defense mechanisms have demonstrated their usefulness to practitioners

72 and theoreticians. The concept of defense is vague as each individual views it differently.

73 However, the number of recognized defense mechanisms has been increasing over the years, and

74 their application has been expanding ${ }^{14}$. In general, defense mechanisms are unconscious

75 psychological strategies individuals use to protect themselves from the anxiety that arises from

76 unacceptable feelings or thoughts ${ }^{15}$. They include denial, projection, isolation,

77 intellectualization, rationalization, displacement, sublimation and reaction formation 16, 17, 18 .

78 Since the inclusion of the structural model of personality (id, ego and superego) in

79 psychoanalytic theory, the concept of defense has been regarded as a mental function and a part

80 of the ego ${ }^{16,19,20}$. Defense mechanisms are significant predictors of several variables, including

81 personality traits, identity status, psychopathology, and the development of the ego ${ }^{21,22}$. Without

82 them, the conscious mind would be more vulnerable to negatively charged emotional input 23,24 ,

$8325,26,27,28$. Valliant proposes the existence of four types of defenses: psychotic defenses,

84 immature defenses, neurotic defenses, and mature defenses 16, 29. According to psychoanalytic

85 theory, defense mechanisms are, in short, unconscious strategies aimed to decrease or eliminate

86 anxiety due to potentially harmful or unacceptable stimuli; they may or may not be associated

87 with a specific behavior 30, 31. After a physical injury, redness, tenderness, and swelling

88 immobilize a fracture so it can heal. Similarly, to physical illnesses, the symptoms of mental

89 illnesses, such as depression or anxiety, are a product of homeostatic struggles with adapting to

90 life ${ }^{32}$.

91 Any individually perceived stress can provoke psychological defense mechanisms $30,31$.

92 Perceived stress and tension resulted from government-enforced quarantines and lockdowns,

93 social distancing, and other preventive measures associated with the COVID-19 pandemic can 
94 provoke psychological defense mechanisms ${ }^{33}$. According to psychoanalytic theory, a defense

95 mechanism is an unconscious or automatic psychological strategy that aims to lessen anxiety

96 arising from intolerable or potentially threatening impulses ${ }^{34}$. It has been theorized that the

97 defense mechanisms employed in response to the COVID-19 pandemic are more likely to be

98 immature, as anxiety over the pandemic can be temporarily relieved by altering painful ideas or

99 distorting one's external reality ${ }^{31,33}$. For example, disavowal is a common defense mechanisms

100 that can be used to protect an individual from something they cannot cope with ${ }^{33}$. Usually,

101 stressors restore internal homeostasis by generating adaptive behavioral and physiological

102 responses ${ }^{35}$. However, when those stressors are uncontrollable, severe, or prolonged, they can

103 lead to negative health consequences, such as post-traumatic stress disorder $35,36,37,38$. The

104 underpinning substrates of defense mechanisms include a collection of mental processes that

105 manipulate or in some other way reject reality to protect an individual from overwhelming

106 feelings of stress and anxiety and from negative stimuli. This can lead to a balance between

107 subjectivity and reality 34,39 . This kind of mechanism protects against internally generated

108 threatening wishes and desires and from external, potentially harmful threats. It can therefore

109 shield the integrity of the ego (mind) and maintain an individual's self-schema ${ }^{34}$. Adjustment

110 after trauma includes resilience and coping with conscious and unconscious content ${ }^{33}$.

111 While living through this worldwide health crisis, individuals have employed a variety of explicit

112 and implicit emotional coping strategies to alleviate the impact of pandemic-related

113 psychological distress ${ }^{40,41}$. These defense mechanisms might lead to coping and adjustment

114 (healthy) behavior or unhealthy (pathological) behavior, which can have serious sequelae.

115 Whether a defense mechanism leads to healthy or unhealthy behavior depends on the

116 environment in which the mechanism is utilized ${ }^{41}$. Individuals' responses to stressful situations 
117 might fall under one of the four categories of defense mechanisms; these responses can improve

118 resilience and protect the ego from internal conflicts ${ }^{34}$. This article serves as a narrative review

119 has two emphases of interest: (1) the assessment of the negative influences of the COVID-19

120 pandemic on the psychological well-being and how the psychological distress, due to the

121 pandemic, would affect and manipulate human's psychological behavior; and (2) the importance

122 of investigating different psychological defense mechanisms that play a critical role in mitigating

123 psychological distress and utilizing mechanisms that promotes mental resilience, in addition, the

124 evaluation of how adjustment mechanisms and coping strategies can protect peoples from

125 internal and external threat.

126

127 Review methodology

128 Literature review was done on PubMed on the relationship of COVID-19 pandemic and defense 129 mechanism, using the keywords "COVID-19" OR "pandemic"; "COVID-19 pandemic," AND

130 "Defense mechanism" OR "Self-mechanism." It also examines evidence of the role of adaptive

131 defense mechanisms in protecting people from psychological distress due to stressful life events,

132 such as the COVID-19 pandemic.

\section{Pathologic defenses}

134 Pathologic defenses, also called psychotic defenses, are mainly characterized by an evident break 135 with objective reality ${ }^{32}$. They permit the individual to restrict conflicts to the external world. In

136 other words, they involve reorganizing external experiences to reduce the need to adjust to

137 reality. Individuals who use these mechanisms may appear irrational or psychotic to others; these

138 defenses represent the most dysregulated level of adjustment and are characterized by a failure to

139 contain the defensive reaction to stressors, leading to a break with objective reality ${ }^{42,}{ }^{43}$. 
140 Pathologic defenses are common in individuals with post-traumatic stress disorder PTSD) and

141 may include delusional projections and psychotic distortion or denial. These mechanisms are

142 common in three situations: in dreams, in cases of psychosis, and among young children. To

143 breach them, neuroleptics, or waking the dreamer, are needed to alter the brain ${ }^{30}$. Psychotic

144 defense mechanisms are an issue in the study of personality and of schizophrenic and bipolar

145 disorders ${ }^{43}$.

146 Immature defenses

147 Immature defenses mainly comfort the user; however, they annoy the observer ${ }^{32}$. These defenses

148 reduce feelings of anxiety and distress generated by perceived harmful impulses or an

149 unacceptable reality. Individuals using such defenses may display outbursts of behavior or

150 emotions that may be socially inappropriate. The goal of such outbursts is to mask undesirable

151 underlying feelings or thoughts. The twelve types of immature defenses are projection, isolation

152 of affect, dissociation, splitting, rationalization, devaluation, denial, acting out, autistic fantasies,

153 somatization, passive-aggression, and displacement. They are used to suppress emotional

154 awareness and can contribute to psychopathology ${ }^{44}$. These kinds of defenses are common

155 among adolescents, substance abusers, and individuals with personality disorders or brain injury.

156 Those defenses rarely respond to verbal interpretations on their own ${ }^{30}$.

157 Immature defenses are a factor in treating patients with depression. One study found that

158 tracking the use of such defenses during a two-year follow-up period led to a significant decrease

159 in the need for treatment compared to a control group ${ }^{45,46}$. Another paper notes that in patients

160 who experienced symptomatic recovery, the use of immature defenses declined significantly,

161 while no change was observed in neurotic or mature defenses ${ }^{47}$. The most common immature

162 defense in individuals with depression is projection ${ }^{45}$, 48 . Immature defenses can be breached in 
163 several ways. The first is confrontation, which is usually done by a group of supportive peers or

164 in focused psychotherapy ${ }^{30,49}$. Therefore, during the COVID-19 pandemic and other difficult

165 times, it is vital to stay connected to support systems. The second approach involves rendering

166 the individual less anxious or lonely by improving intrapsychic competence through empathy.

167 The third approach is to improve brain function, such as by relieving normal-pressure

168 hydrocephalus $30,50,51$.

169 Neurotic defenses

170 Neurotic defenses keep all potentially threatening feelings, memories, ideas, wishes, or fears

171 outside an individual's awareness ${ }^{30}$. They can be used to manage or control an external violent

172 and vulnerable environment and to avoid underlying feelings of anxiety and guilt. This kind of

173 defense offers short-term rewards for dealing with stressful events; however, long-term use of

174 such defenses can lead to a wide range of relationship challenges. These defenses manifest

175 clinically as compulsions, somatizations, phobias, and amnesia. Unlike immature defenses ${ }^{30}$,

176 neurotic defenses typically make the user more uncomfortable than the observer ${ }^{30,}{ }^{32}$. Neurotic

177 defenses can commonly be breached with psychotherapy ${ }^{30}$.

178 Evidence indicates that neurotic defenses play a critical role in mediating the psychological

179 effects of the COVID-19 pandemic. Several dynamic coping strategies that fall into this category

180 have been employed to buffer individuals from the effects of the pandemic. One example is

181 repression. This may involve avoiding conscious awareness of or exposure to information about

182 the dangers of COVID-19. However, this knowledge continues to influence peoples' behavior; it

183 does not simply vanish. Another example is dissociation, such as when healthcare workers try to

184 dissociate or disconnect from negative experiences, memories, thoughts, sensations, and even

185 their identities during the pandemic. A third example is reaction formation, which is when 
186 individuals express the opposite of their real feelings and emotions. This kind of transformation

187 of emotions may help promote mental resilience. Another example of this kind of defense

188 mechanism is displacement, such as when a patient infected with COVID-19 expresses anger and

189 blames family members or others for their illness.

\section{Mature defenses}

191 Mature defense are conscious processes that boost feelings of control and augment positive 192 emotions; they result in optimal adaptation to stressors ${ }^{32}$. These defenses typically enhance 193 gratification and increase one's conscious awareness of their own ideas and feelings and their 194 consequences ${ }^{30,32}$. They can also help individuals balance conflicting motivations ${ }^{21}$. Examples 195 of mature defenses include altruism, sublimination, suppression, and humor. Even though humor 196 seems to reflect dissociation and denial, it, like meditation, can help shift the body to 197 parasympathetic calmness from autonomic sympathetic agitation ${ }^{30}$. Mature defenses increase 198 with age ${ }^{30,52}$, in contrast to PTSD, which usually decreases in severity with age ${ }^{30}$. Coping 199 responses to stress can be broadly divided into three categories: 1) voluntarily asking for help 200 from appropriate persons (such as mobilizing social supports), 2) voluntary strategies (such as 201 gathering information and anticipating danger) 34, 53, and 3) unconscious homeostatic 202 mechanisms such as fever or leukocytosis; these are involuntary physical responses that help 203 reduce the effects of stress ${ }^{34}$.

\section{Adaptive defense mechanisms and the COVID-19 pandemic}

205 Research has demonstrated the role of adaptive defense mechanisms in protecting people from 206 psychological distress due to stressful life events, such as the COVID-19 pandemic. Defense 207 mechanisms have played an important role in mitigating psychological distress during the 208 COVID-19 pandemic, and utilizing defense mechanisms promotes mental resilience $54,55$. 
209 Walker et al. identified several psychological defense mechanisms employed during the COVID-

21019 pandemic, including denial, hypochondriasis, altruism, sublimation, and humor. Of these,

211 altruism, sublimination, and humour are highly adaptive defenses that maximize gratification and

212 help individuals become more aware of their own feelings and ideas and their consequences ${ }^{54}$.

213 It is important to consider defense mechanisms within the larger structure of stress and coping ${ }^{32}$.

214 A recent meta-analysis of stressful and traumatic experiences during the COVID-19 pandemic 215 found high rates of anxiety (31.9\%), depression (33.7\%), and stress (29.6\%) ${ }^{56}$. Such

216 psychological impacts can lead to various acute anxiety reactions, which may be accompanied by

217 maladaptive behavior or immature defense mechanisms. The pandemic and lockdowns have

218 impacted young children and adolescents more significantly than adults due to their immature

219 emotional and social development. Younger children are more likely to experience clinginess

220 and the fear of COVID-19 affecting family members; in older children, impacts may take the

221 form of inattention or constant inquiries about COVID-19. Increased irritability, inattention, and

222 clinginess have been observed in children of all age groups ${ }^{57,58}$.

223 During any global pandemic, the use of defense mechanisms should be continuously observed

224 since the capability to properly utilize a variety of defenses in perplexing environments is

225 associated with mental and psychological health. Furthermore, the use of immature defenses is a

226 pathogenic predictor for psychiatric conditions ${ }^{59}$. Distress increases when individuals rely less

227 on adaptive defense mechanisms ${ }^{59}$.

228 Giuseppe et al. note that the overall defensive functioning of participants during the first week of

229 lockdown in the ongoing pandemic fell within normal neurotic ranges ${ }^{55}$. However, in individuals

230 experiencing distress or depression, overall defensive functioning correlated negatively with the

231 severity of symptoms ${ }^{55}$. The use of immature defenses correlated positively with more severe 
232 symptoms ${ }^{55}$. Moreover, higher defensive functioning (of mature defenses) correlated negatively

233 with levels of depression and post-traumatic stress ${ }^{54,55}$. However, when immature defense

234 mechanisms such as neurotic behavior are used, the risk of developing pathological personality

235 traits increases ${ }^{60}$. Despite this, neurotic defenses play a role in managing virus-related anxiety ${ }^{54}$

$236,59,61$. Evidence indicates that neurotic defenses play a critical role in mediating the psychological

237 effects of the COVID-19 pandemic. Several dynamic coping strategies that fall into this category

238 have been employed to buffer individuals from the effects of the pandemic. One example is

239 repression. This may involve avoiding conscious awareness of or exposure to information about

240 the dangers of COVID-19. However, this knowledge continues to influence peoples' behavior; it

241 does not simply vanish. Another example is dissociation, such as when healthcare workers try to

242 dissociate or disconnect from negative experiences, memories, thoughts, sensations, and even

243 their identities during the pandemic. A third example is reaction formation, which is when

244 individuals express the opposite of their real feelings and emotions. This kind of transformation

245 of emotions may help promote mental resilience. Another example of this kind of defense

246 mechanism is displacement, such as when a patient infected with COVID-19 expresses anger and

247 blames family members or others for their illness.

248 The most common depressive symptoms during the pandemic include depression, anxiety, and

249 PTSD 55, 62, 63. In an Italian cohort, different levels of defenses were related to varying degrees of

250 adaptation to stress ${ }^{55}$. In addition, emotional regulation is of utmost importance during stressful

251 times, particularly among vulnerable groups, such as patients suffering from physical or mental

252 illnesses $55,64,65,66,67,68,69$. One study of defense mechanisms and coping strategies found that

253 coping mechanisms, a positive attitude, and mature defense mechanisms negatively correlated 
254 with perceived stress ${ }^{70}$. In addition, life satisfaction, or the perception of having a full, 255 meaningful life ${ }^{70,71}$, is associated with lower stress levels and more active adaptive coping ${ }^{70,72 .}$

256 All these findings can be applied to responses to the COVID-19 pandemic. During this crisis,

257 people have sought help from experienced personnel, prepared by complying with precautionary

258 measures, and tried to fight COVID-19 if they became infected. Higher defensive functioning is

259 associated with lower levels of depression, anxiety, and post-traumatic stress symptoms. On the

260 other hand, as stated above, Italians with poorer adaptive defense functioning experienced higher

261 levels of stress during the first week of lockdown ${ }^{55}$.

\section{Recommendations}

263 Individuals can take some steps to enhance their psychological defensive responses to COVID-

26419 or any similar pandemic. For example, physical exercise modulates the immune system;

265 cytokines, both pro- and anti-inflammatory, are released during and after an exercise session.

266 Exercise also increases cell recruitment and lymphocyte circulation ${ }^{73}$. Multiple studies have

267 found that regular moderate exercising helps reduce the frequency of upper respiratory tract

268 infections (URTIs) ${ }^{74,75,76,77}$. This is because exercise helps stimulate cellular immunity ${ }^{73,78,79}$.

269 Adequate sleep patterns also help modulate the adaptive immune response ${ }^{74,80}$. Therefore,

270 during the pandemic, it is vital to maintain an overall healthy lifestyle while also adhering to all

271 recommended precautionary measures to decrease one's chances of becoming infected.

272 Seeking help when needed is also extremely important. Thanks to telemedicine, help is now

273 within nearly everyone's reach. Although there are many challenges to telemedicine, during the

274 pandemic, its positive features outweigh the negative. Moreover, offering continuous support

275 within communities and families is of utmost importance during such difficult times. Finally, 
276 individuals must cope with their current circumstances in healthy and productive ways by

277 developing a more conscious relationship with reality.

278

279 Conclusion

280 The COVID-19 pandemic has had a wide range of negative physical and mental impacts. This

281 review has highlighted some of the psychological defence mechanisms individuals use to deal

282 with the pandemic. Defense mechanisms are unconscious strategies utilized to protect

283 individuals from anxiety or stress arising from intolerable stimuli. They are a collection of

284 mental processes that defend against overwhelming feeling of stress and threatening stimuli and

285 promote mental resilience. Individuals' responses to stressful situations might fall under one of

286 the four types of defense mechanisms (pathologic, immature, neurotic, and mature); these

287 defense mechanisms can improve resilience and protect the ego from internal conflict.

288 Individuals who employ higher levels of defensive functioning, such as mature defenses, are less

289 likely to develop psychiatric problems or maladaptive behaviors. Individuals who use immature

290 defense mechanisms, such as neurotic defenses, have a higher risk of developing pathological

291 personality traits.

292 Individuals can take steps to enhance their own defense mechanism responses to COVID-19 or 293 any similar pandemic. For example, physical activity modulates the immune system.

294 Psychosocial interventions play a critical role in reducing the risk of developing psychiatric and

295 personality disorders due to a crisis or pandemic. Individuals employ a wide variety of explicit

296 and implicit emotional coping strategies to reduce the psychologically distressing impacts of the

297 pandemic ${ }^{41}$. The healthy, productive use of defense mechanisms can encourage coping and

298 healthy behavior that helps counter psychological distress. 
302 Acknowledgment: All aauthors declare no grant support for this work.

303 Conflicts of interest: Authors declare no conflict of interest

304 Ethical approval: Not required

305

306

307

308

309

310

311

312

313

314

315

316

317

318

319

320

321 


\section{References}

326 1. Holmes EA, O’Connor RC, Perry VH, Tracey I, Wessely S, Arseneault L, et al. Multidisciplinary research priorities for the COVID-19 pandemic: a call for action for mental health science. Lancet Psychiatry. 2020;7:547-560.

2. Li S, Wang Y, Yang Y, Lei X, and Yang Y. Analysis of influencing factors of anxiety and emotional disorders in children and adolescents during home isolation during the epidemic of novel coronavirus pneumonia. Chin. J. Child Heal 2020, 1-9.

3. Cao W, Fang Z, Hou G, Han M, Xu X, Dong J, et al. The psychological impact of the COVID-19 epidemic on college students in China. Psychiatry Res. 2020 May;

4. Carragher N, Krueger RF, Eaton NR, Slade T. Disorders without borders: Current and future directions in the meta-structure of mental disorders. Social Psychiatry and Psychiatric Epidemiology. 2015; 50(3):339-350.

5. De Berardis D, Fornaro M, Valchera A, Cavuto M, Perna G, Di Nicola M, et al. Eradicating Suicide at Its Roots: Preclinical Bases and Clinical Evidence of the Efficacy of Ketamine in the Treatment of Suicidal Behaviors. Int J Mol Sci. 2018; 19(10):2888. doi: 10.3390/ijms19102888. PMID: 30249029; PMCID: PMC6213585.

6. Thombs BD, Bonardi O, Rice DB, Boruff JT, Azar M, He C, et al. Curating evidence on mental health during COVID-19: A living systematic review. J Psychosom Res. 
2020:110113. pmid:32354463

346

347

348

349

350

351

352

353

354

355

356

357

358

359

360

361

362

363

364

365

366
7. Olashore AA, Akanni OO, Fela-Thomas AL, \& Khutsafalo K. The psychological impact of COVID-19 on health-care workers in African Countries: A systematic review. Asian Journal of Social Health and Behavior. 2021: 4(3), 85.

8. Bahar Moni AS, Abdullah S, Bin Abdullah MFIL, Kabir MS, Alif SM, Sultana F, et al. Psychological distress, fear and coping among Malaysians during the COVID-19 pandemic. PLoS One. 2021; 16(9):e0257304. doi: 10.1371/journal.pone.0257304. PMID: 34506576; PMCID: PMC8432783.

9. Xiao H, Zhang Y, Kong D, Li S, Yang N. The Effects of Social Support on Sleep Quality of Medical Staff Treating Patients with Coronavirus Disease 2019 (COVID-19) in January and February 2020 in China. Med Sci Monit. 2020; 26:e923549. pmid: 32132521

10. Lin CY. Social reaction toward the 2019 novel coronavirus (COVID-19). Soc Health Behav 2020; 3:1.

11. Labrague LJ, de los Santos J. Fear of COVID-19, psychological distress, work satisfaction and turnover intention among frontline nurses. J Nurs Manag 2021; 29:395403.

12. Rajabimajd N, Alimoradi Z, \& Griffiths MD. Impact of COVID-19-related fear and anxiety on job attributes: A systematic review. Asian Journal of Social Health and Behavior, 2021: 4(2), 51-55.

13. Brooks SK, Webster RK, Smith LE, Woodland L, Wessely S, Greenberg N, et al. Rapid Review The psychological impact of quarantine and how to reduce it: rapid review of the evidence. Lancet 2020;395:912-20.

14. Gabbard GO. Psychoanalytic Thinking in Contemporary Psychiatry. Comprehensive Text 
368 Book of Psychiatry Kaplan and Sadock; 1995

369 15. Bailey R, Pico J. Defense Mechanisms. StatPearls Publishing; 2021

370 16. Cramer P. Understanding defense mechanisms. Psychodyn Psychiatry 2015; 43:523-52.

371 17. Thomä H. Psychoanalytic theory and therapy of neurotic anxieties. Psyche (Stuttg).

372 $1995 ; 49: 1043-67$

373

374

18. Freud A. The ego's defensive operations considered as an object of analysis. Ego Mech Def. $1936 ; 1992$.

19. Freud S. Inhibitions, symptoms and anxiety. Psychoanal. Q. 1936;5:1-28

20. Frederickson JJ, Messina I, Grecucci A. Dysregulated anxiety and dysregulating defenses: Toward an emotion regulation informed dynamic psychotherapy. Front. Psychol. 2018;9:2054.

21. Cramer P. The Development of Defense Mechanisms during the Latency Period. J Nerv Ment Dis. 2018;206:286-9.

22. Sullivan HS. The Interpersonal Theory of Psychiatry. Routledge; Abingdon, UK: 2013.

382 23. Busch FN, Milrod BL, Singer MB, Aronson AC. Manual of Panic Focused

383 Psychodynamic Psychotherapy-Extended Range. Routledge; Abingdon on Thames, UK: 2011.

25. Finlay-Jones R, Brown GW. types of stressful life event and the onset of anxiety and depressive disorders. Psychol Med. $1981 ; 11: 803-15$. 
391

392

393

Res Ther. 1993 ;31:57-62.

27. Rapee RM. Perceived threat and perceived control as predictors of the degree of fear in physical and social situations. J Anxiety Disord. 1997;11:455-61.

28. Shaver P, Schwartz J, Kirson D, O’Connor C. Emotion knowledge: Further exploration of a prototype approach. J Pers Soc Psychol. 1987;52(6).

29. Vaillant, G. E. (1992). Ego mechanisms of defense: A guide for clinicians and researchers. Washington, DC: American Psychiatric Press.

30. Vaillant GE. Involuntary coping mechanisms: A psychodynamic perspective. Dialogues Clin Neurosci . 2011;13(3):366-70.

31. Walker G, McCabe T. Psychological defence mechanisms during the COVID-19 pandemic: A case series. Eur J Psychiatry 2021;35:41-5.

32. Żechowski C. Theory of drives and emotions - from Sigmund Freud to Jaak Panksepp. Psychiatr Pol. 2017;51:1181-1189.

33. Marčinko D, Jakovljević M, Jakšić N, Bjedov S, Mindoljević Drakulić A. The Importance of Psychodynamic Approach during COVID-19 Pandemic. Psychiatr Danub. $2020 ; 32: 15-21$.

34. Vaillant GE. Involuntary coping mechanisms: A psychodynamic perspective. Dialogues Clin Neurosci 2011;13:366-70.

35. Cooper MA, Clinard CT, Morrison KE. Neurobiological mechanisms supporting experience-dependent resistance to social stress. Neuroscience 2015; 1-14.

36. Abelson JL, Khan S, Liberzon I, Young EA. HPA axis activity in patients with panic disorder: Review and synthesis of four studies Depress Anxiety 2007; 66-76.

37. Meewisse ML, Reitsma JB, De Vries GJ, Gersons BPR, Olff M. Cortisol and post- 
414

415

416

417

418

419

420

421

422

423

424

425

426

427

428

429

430

431

432

433

434

435

436 traumatic stress disorder in adults: Systematic review and meta-analysis. Br J Psychiatry $2007 ; 387-92$.

38. Heim C, Newport DJ, Mletzko T, Miller AH, Nemeroff CB. The link between childhood trauma and depression: Insights from HPA axis studies in humans.

Psychoneuroendocrinology $2008 ; 33: 693-710$.

39. American Psychiatric Association. DSM. History of the Manual. [Last accessed on 2013, Jul 1]. Available from: http://www.psychiatry.org/practice/dsm/dsm-history-of-themanual.

40. Gyurak A, Gross JJ, Etkin A. Explicit and implicit emotion regulation: A dual-process framework. Cogn Emot 2011; 400-12.

41. Rice TR, Hoffman L. Defense Mechanisms and Implicit Emotion Regulation: A Comparison of a Psychodynamic Construct with One from Contemporary Neuroscience. J Am Psychoanal Assoc 2014; 693-708.

42. Vaillant GE. Theoretical Hierarchy of Adaptive Ego Mechanisms: A 30-Year Follow-up of 30 Men Selected for Psychological Health. Arch Gen Psychiatry 1971;24:107-18.

43. Berney S, de Roten Y, Beretta V, Kramer U, Despland JN. Identifying Psychotic Defenses in a Clinical Interview. J Clin Psychol 2014;70:428-39.

44. Costa RM, Brody S. Immature psychological defense mechanisms are associated with greater personal importance of junk food, alcohol, and television. Psychiatry Res. 2013;209:535-9.

45. Bond M. Empirical studies of defense style: Relationships with psychopathology and change [Internet]. Vol. 12, Harvard Review of Psychiatry. Harv Rev Psychiatry 2004; $263-78$. 
46. Akkerman K, Lewin TJ, Carr VJ. Long-term changes in defense style among patients recovering from major depression. J Nerv Ment Dis 1999;187:80-7.

47. Akkerman K, Carr V, Lewin T. Changes in ego defenses with recovery from depression. J Nerv Ment Dis 1992;180:634-8.

48. Spinhoven P, Kooiman CG. Defense style in depressed and anxious psychiatric outpatients: An explorative study. J Nerv Ment Dis 1997;185:87-94.
50. Perry JC, Beck SM, Constantinides P, Foley JE. Studying Change in Defensive

53. Tovote P, Fadok JP, Lüthi A. Neuronal circuits for fear and anxiety. Nat. Rev. Neurosci. 2015;16:317.

54. Walker G, McCabe T. Psychological defence mechanisms during the COVID-19 pandemic: A case series. Eur J Psychiatry. 2021 ;35:41-45.

55. Di Giuseppe M, Zilcha-Mano S, Prout TA, Perry JC, Orrù G, Conversano C. Psychological Impact of Coronavirus Disease 2019 Among Italians During the First Week of Lockdown. Front Psychiatry 2020;11:1-5

56. Salari N, Hosseinian-Far A, Jalali R, Vaisi-Raygani A, Rasoulpoor S, Mohammadi M, et 
al. Prevalence of stress, anxiety, depression among the general population during the

461 COVID-19 pandemic: A systematic review and meta-analysis Globalization and Health.

462 $2020 ; 1-11$. lockdown on mental health of children and adolescents: A narrative review with recommendations. Psychiatry Res. 2020;293:113429.

58. Viner RM, Russell SJ, Croker H, Packer J, Ward J, Stansfield C, et al. School closure and management practices during coronavirus outbreaks including COVID-19: a rapid systematic review. The Lancet Child and Adolescent Health. 2020; 397-404. K, et al. Identifying Predictors of Psychological Distress During COVID-19: A Machine Learning Approach. Front Psychol. 2020 5;11:586202. Mechanisms and Pathological Personality Characteristics. Rev Colomb Psiquiatr (Engl Ed). $2019 ; 48: 232-243$.

61. Di Giuseppe M, Perry JC, Lucchesi M, Michelini M, Vitiello S, Piantanida A, et al. Preliminary Reliability and Validity of the DMRS-SR-30, a Novel Self-Report Measure Based on the Defense Mechanisms Rating Scales. Front Psychiatry 2020;11:870.

62. Perry JC, Bond M. Change in defense mechanisms during long-term dynamic psychotherapy and five-year outcome. Am J Psychiatry 2012;169:916-25.

63. Babl A, grosse Holtforth M, Perry JC, Schneider N, Dommann E, Heer S, et al. Comparison and change of defense mechanisms over the course of psychotherapy in 482 patients with depression or anxiety disorder: Evidence from a randomized controlled trial. 
J Affect Disord 2019;252:212-20.

484

485

486

487

488

64. Zilcha-Mano S. Toward Personalized Psychotherapy: The Importance of the TraitLike/State-Like Distinction for Understanding Therapeutic Change. Am Psychol. $2020 ; 56-62$

65. Boldrini T, Lo Buglio G, Giovanardi G, Lingiardi V, Salcuni S. Defense mechanisms in adolescents at high risk of developing psychosis: An empirical investigation. Res Psychother Psychopathol Process Outcome 2020;23:4-15. A

66. Di Giuseppe M, Perry JC, Conversano C, Gelo OCG, Gennaro A. Defense mechanisms, gender, and adaptiveness in emerging personality disorders in adolescent outpatients. $\mathbf{J}$ Nerv Ment Dis 2020;208:933-41.

67. Martino G, Caputo A, Schwarz P, Bellone F, Fries W, Quattropani MC, et al. Alexithymia and inflammatory bowel disease: A systematic review. Front Psychol $2020 ; 11$.

68. Martino G, Caputo A, Vicario CM, Catalano A, Schwarz P, Quattropani MC. The Relationship Between Alexithymia and Type 2 Diabetes: A Systematic Review. Frontiers in Psyc. 2020;124-130

69. Nam J-W, Ha JH, Choi E, Park D-H, Ryu S-H. Mature Defense Mechanisms Affect Successful Adjustment in Young Adulthood-Adjustment to Military Service in South Korea. Psychiatry Investig 2019 ;16(7):484-90.

70. Gori A, Topino E, Di Fabio A. The protective role of life satisfaction, coping strategies and defense mechanisms on perceived stress due to COVID-19 emergency: A chained mediation model. PLoS One $2020 ; 15(11)$.

71. Grecucci A., Theuninck A., Frederickson J., Job R. Emotion Regulation: Processes, 
506 Cognitive Effects and Social Consequences. Nova Publishers; Hauppauge, NY, USA:

507 2015. Mechanisms of social emotion regulation: From neuroscience to psychotherapy;

$508 \quad$ pp. $57-84$.

509 72. Hooker SA, Masters KS, Park CL. A meaningful life is a healthy life: A conceptual

510 model linking meaning and meaning salience to health. Rev Gen Psychol 2018;22:11-

51124.

512 73. 6da Silveira MP, da Silva Fagundes KK, Bizuti MR, Starck É, Rossi RC, de Resende e

513 Silva DT. Physical exercise as a tool to help the immune system against COVID-19: an

$514 \quad$ integrative review of the current literature. Clinical and Experimental Medicine 2021;

$515 \quad 15-28$.

516 74. Larenas-Linnemann D, Rodríguez-Pérez N, Arias-Cruz A, Blandón-Vijil MV, Del Río-

517 Navarro BE, Estrada-Cardona A, et al. Enhancing innate immunity against virus in times

518 of COVID-19: Trying to untangle facts from fictions. World Allergy Organ J. 2020

$519 \quad ; 13: 100476$

520 75. Matthews CE, Ockene IS, Freedson PS, Rosal MC, Merriam PA, Hebert JR. Moderate to

521 vigorous physical activity and risk of upper-respiratory tract infection. Med Sci Sports

$522 \quad$ Exerc 2002;34:1242-8.

523 76. Fondell E, Lagerros YT, Sundberg CJ, Lekander M, Bölter O, Rothman KJ, et al.

524 Physical activity, stress, and self-reported upper respiratory tract infection. Med Sci

$525 \quad$ Sports Exerc 2011;43:272-9.

526 77. Nieman DC, Henson DA, Austin MD, Sha W. Upper respiratory tract infection is

527 reduced in physically fit and active adults. Br J Sports Med 2011;45:987-92.

528 78. Pedersen BK, Hoffman-Goetz L. Exercise and the immune system: Regulation, 
529 integration, and adaptation. Physiological Reviews. 2000; 1055-81.

530 79. Bajpai G, Nahrendorf M. Infectious and lifestyle modifiers of immunity and host

531 resilience. Immunity. 2021;54:1110-1122.

532 80. Spiegel K. Effect of Sleep Deprivation on Response to Immunizaton. JAMA J Am Med $533 \quad$ Assoc 2002;288:1471-a-1472.

535

536

537

538 